\title{
COMPUTATIONAL FLUID DYNAMICS INVESTIGATION ON THE USE OF HEAT SHIELDS FOR THERMAL MANAGEMENT IN A CAR UNDERHOOD
}

\author{
S.Y. Lam ${ }^{1}$, N.H. Shuaib ${ }^{2}$, H. Hasini ${ }^{1}$ and N. A. Shuaib ${ }^{3}$ \\ ${ }^{1}$ Centre for Advanced Computational Engineering (CACE), \\ University Tenaga Nasional (UNITEN), MALAYSIA. \\ ${ }^{2}$ TNB Research Sdn. Bhd., MALAYSIA. \\ ${ }^{3}$ School of Manufacturing Engineering, University Malaysia Perlis (UNIMAP), \\ MALAYSIA \\ Email: norshahhafeez@tnbr.com.my
}

\begin{abstract}
Temperature variations inside a car underhood are largely controlled by the heat originating from the engine block and the exhaust manifold. Excessive temperatures in the underhood can lead to the faster deterioration of engine components and may affect the thermal comfort level inside the passenger cabin. This paper presents computational fluid dynamics investigations to assess the performance of a heat shield in lowering the peak temperature of the engine components and firewall in the underhood region of a typical passenger car. The simulation used the finite volume method with the standard $\mathrm{k}-\varepsilon$ turbulence model and an isothermal model for the heat transfer calculations. The results show that the heat shield managed to reduce the peak temperature of the engine components and firewall by insulating the intense heat from the engine block and exhaust and regulating the airflow inside the underhood region.
\end{abstract}

Keywords : Heat shield; car underhood; CFD; numerical simulations.

\section{INTRODUCTION}

In current vehicle design and development, the best design is normally achieved through design-evaluate-redesign cycle. In terms of passenger cars, the car underhood has always been the focus of these cycles as car manufacturers search for an optimal thermal condition to improve their vehicle's engine management system. Thermal management in a car underhood is particularly important for improving passenger comfort and reducing the thermal wear of engine components, as well as helping the design meet new energy efficiency and emission requirements under normal working conditions. Besides, thermal management in a car underhood serves as fire prevention, to ensure that the design of the passenger car complies with the fire safety standards.

The firewall of a car is located between the underhood and the passenger cabin. It serves as a heat insulator for the passenger cabin from the intense heat of the underhood and as protection during the incidence of fire or smoke. The time taken for the heat to dissipate from the heat source, such as the engine block or the exhaust manifold, towards the passenger cabin is a function of the geometrical parameters of the underhood. Ideally, the location of the heat source should be blocked or positioned as far away as possible from the firewall. However, the blockage of these heat sources may result in the impediment of hot air circulation, which in turn increases the temperature of other engine components, resulting in the overheating of the engine. 
Several approaches have been proposed to perform computational fluid dynamics (CFD) simulations within the underhood environment. Davis et al. (1993) describes the impact of conducting underhood airflow simulations on the cooling design and development process. Fellague et al. (1994) provides a correlation for front end cooling airflow studies. Hsu and Schwartz (1995) described an approach wherein local studies of under-body components are coupled by transferring boundary conditions to the local models. Srinivasan et al. (1999) has demonstrated the use of an adaptive Cartesian based mesh for accurate front-cooling airflow predictions, and the feasibility of building local models of the underhood to understand the thermal environments. Two types of inlet boundary were modeled to represent the combination of ram air with a cooling fan, and air flow beneath a vehicle's front end. An outlet boundary was also modeled to represent the flow exiting downstream into the under body of the car. Coupled radiation/convection simulations were performed to obtain the complete airflow and thermal map of the underhood. Yang et al. (2004) simulated an idle vehicle with just the cooling fans operating. Cooling airflow rates at idling conditions and hot air re-circulation were modeled in this work. Cheng et al. (2000) suggested conjugate heat transfer through an interface with ambient air coupled with radiation, which was modeled to predict the temperature distribution inside an engine component. A standard $\mathrm{k}-\varepsilon$ model with a wall function was selected to model turbulence, and a discrete ordinate (DO) radiation model was selected to simulate the heat transfer from the heat source to other components. Gray radiation surface-to-surface in an optical thin medium was assumed in this model.

Srivanesan et al. (2004) explained that when a vehicle is in motion the airflow stream beneath the front end also becomes part of the underhood convection mechanism. The outer surface of the exhaust system will become the primary heat source to the engine compartment, and the temperature in the exhaust manifolds and muffler can range from $785 \mathrm{~K}$ to $952 \mathrm{~K}$ when the engine is outputting close to maximum capacity with stoichiometric air fuel ratio based combustion. The monitoring of components at risk of thermal failure is essential, and appropriate thermal protection could be achieved by the insertion of a heat shield between the exhaust and components or an innovative airflow management technique that increases the convection around the components. The physical domain used for the computation starts from the cooling module and extends downstream to the under-dash panel/A-pillar of the vehicle. All engine components are enclosed within the volume of the car underhood, which forms a closed volume with multiple closed volumes contained inside it. Mesh generation uses interior to boundary (I2B) and shrink wrap techniques that envelop the complex geometry and eliminates the need of "water tight" geometry at the start of grid generation. The standard $\mathrm{k}-\varepsilon$ turbulence model in conjunction with wall functions is used for turbulence closure. Isothermal wall boundary conditions with wall functions are specified at the heat source and a surface-to-surface based radiation model is used to simulate the heat transfer across the car underhood.

Amodeo et al. (2006) performed thermal management on a car underhood using the Lattice-Boltzmann method (LBM). The Lattice-Boltzmann equation (LBE) solvers represent an alternative to the Navier-Stokes solvers, as it does not require a special iterative procedure and achieves mass, momentum, and energy conservation by design. LBE solvers are numerically very efficient and robust, and the increased numerical efficiency allows the handling of lattices with very large numbers of elements (or voxels). In addition, the properties of LBE allow for active elements that interact with the neighboring lattice elements, hence improving treatment of the interaction of the 
fluid flow with the wall surface. The combination of both large lattices and dynamic surface treatments allow the accurate representation of surfaces without the need for geometry simplification. The heat transfer between airflow and heat exchangers is modeled using a one-dimensional tool (1D-tool) and the flow of the fan is simulated with a Multiple Reference Frame (MRF). The heat transfer with this method is predicted with great accuracy. Campos et al. (2007) presented an optimization study and analysis on a cooling duct located in a car underhood. The duct channels air from the side grill and directs it over the transmission for the purpose of cooling the transmission surface and oil. Minimizing the pressure drop across the cooling duct and maximizing the outlet flow velocity magnitude are among the objectives for optimization of a cooling duct. A standard high Reynolds k- $\varepsilon$ with a non-equilibrium wall function was applied, and the SIMPLE solution for pressure-velocity coupling was employed for the simulation. The turbulence intensity is set at $10 \%$, while the length scale is set at $0.025 \mathrm{~m}$ with reference to the wind tunnel data for a full vehicle travelling at $250 \mathrm{kph}$. By coupling the CAD, CFD and optimization software, both design objectives were achieved in addition to good performance and stability.

Wang and Yang (2006) assessed the performance of different frontal grill configurations with a model of airflow across the under-hoof of a minivan. Fascia geometry is found to have a crucial effect on the cooling airflow to the heat exchanger. The turbulent equation is solved using the standard k- $\varepsilon$ model with wall function. The radiator is treated as porous media which yields a drag force to the airflow passing through them. Rather than making numerical predictions for the specific dissipation, the flow is modeled isothermally and the heat exchanger air mass flow rate is recorded. Analysis is conducted based on the mass flow flux, static pressure and streamline near the grill. CFD analysis suggests that the cooling performance of the underhood is sensitive to front end parts, particularly around the lower opening such as the shape and position of the bumper beam and grills. Minimizing blockages to the flow-path and redirecting the flow have distinct rewards on the front-end flow, with flow reorganization providing a pronounced pressure increase and better uniformity for the condenser, radiator and fan module (CRFM) package. Damodaran and Kaushik (2000) split the issues encompassing a car underhood simulation. Front end, underhood and passenger cabin are simulated separately in a conventional way, while the results of each analysis are used to input into the other as boundary conditions, which in turn reduces the accuracy due to the lack of interaction between the two separate models. In most models, air density is assumed to be constant rather than varying with temperature. It may take a relatively long time to build a CFD model if a structured mesh is used due to the grid generation in complex underhood geometry. Therefore, unstructured triangular mesh is adopted to resolve the issue. The inlet temperature at the exhaust is based on the fuel flow rate, while the flow at the manifold inlet values and radiator and condenser heat rejections are required for use as boundary conditions in the CFD model. Vehicle speed and fan RPM were also used as input into the analysis. Combining critical underhood design issues in a single analysis has resulted in a reduction in analysis time and improved accuracy.

Koschan et al. (2006) incorporate thermal imaging in CFD simulation to perform thermal analysis on engine components. Boundary conditions and thermal properties for the simulation are adapted from the thermal image undertaken in real time. In the simulation of vehicle components, the knowledge of certain parameters, e.g. environmental conditions surrounding the vehicle, temperature curves of the components with respect to time, and the materials of the component surfaces and their 
emissivity are required. Environmental parameters like wind direction, humidity and solar radiance, determine the starting ambience of the vehicle.

Based on previous studies, the aim of the underhood simulations or experiments is to maximize both the blockage of the hot heat source and the amount of air recirculation within the underhood. In this paper, the achievement of these two conflicting objectives is proposed by introducing a heat shield at a suitable location inside the underhood of a typical passenger car. The exhaust manifold and engine block are used as the heat sources that interact with the airflow, and the heat is assumed to be dissipated by means of convection and radiation to other engine components. The commercial CFD software FLUENT is used to simulate the airflow and heat transfer inside the underhood. Throughout the simulation, thermal analysis is conducted using the isothermal method where the thermal calculations are performed using constant surface temperatures of the heat sources as the boundary conditions. CFD simulation results are then used to investigate the performance of the proposed heat shield in achieving the abovementioned objectives. This is done by comparing the temperature distribution on the engine components between the base design and the design with the heat shield added.

\section{METHODOLOGY}

\section{Simulation Domain And Mesh}

The simulation was performed with the car model inside a virtual wind tunnel, as shown in Figure 1. As mentioned in the introduction, the analysis presented in this paper will concentrate on the flow behavior and temperature distribution within the underhood region. The car underhood domain and the components inside the domain are shown in Figure 2. The locations and sizes of each component are representative of real components in a typical passenger car, with minor details omitted for the CFD analysis. As shown in Figure 2, the airflow enters the underhood region through two vents at the front of the car. This configuration is denoted as the base design (BD), which will later be used for comparisons with the design with the heat shield added. The mesh inside the underhood region was created using around 900,000 unstructured tetrahedral cells. The mesh configuration is shown in Figure 3. The mesh in the horizontal and vertical planes is taken from the center of the underhood. As depicted, the mesh was generated such that a finer mesh distribution is clustered near the firewall, since those are the regions of concern requiring a more accurate prediction of flow and temperature behavior. As part of the meshing procedure, mesh dependency studies were performed on three different mesh sizes, namely around meshes of 500,000, 900,000 and 1.3 million. The temperature results from the latter two mesh sets are nearly identical, and thus the model with 900,000 is used throughout this study.

To reduce the peak temperature inside the underhood, the inclusion of a heat shield is deemed suitable as it is expected to insulate the engine components from the intense heat around the exhaust manifolds. As shown in Figure 4, the proposed heat shield has a channel that will direct heated air vertically upwards, and away from the exhaust manifold. This will prevent the heat from being channeled directly towards the firewall, thus reducing the peak temperature at the firewall. The proposed heat shields are made of steel plate and have a thickness of $1.5 \mathrm{~mm}$. From this point onwards, to aid the comparison and discussion with the base design (BD), the underhood configuration with heat shield added is referred to as the heat shield design (HSD) 


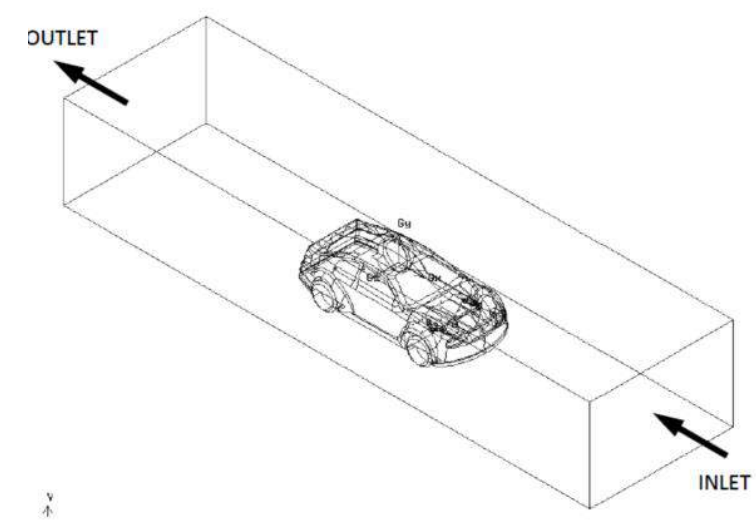

Figure 1. Domain of the car in a virtual wind-tunnel

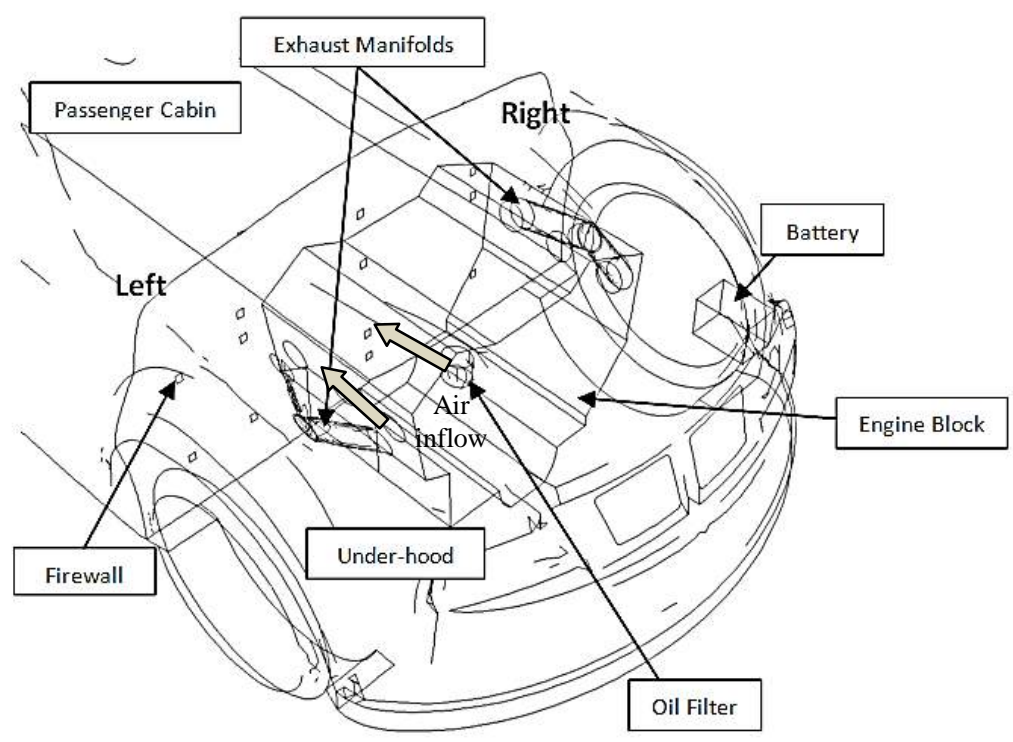

Figure 2. Layout of the car underhood and modeled components (BD)
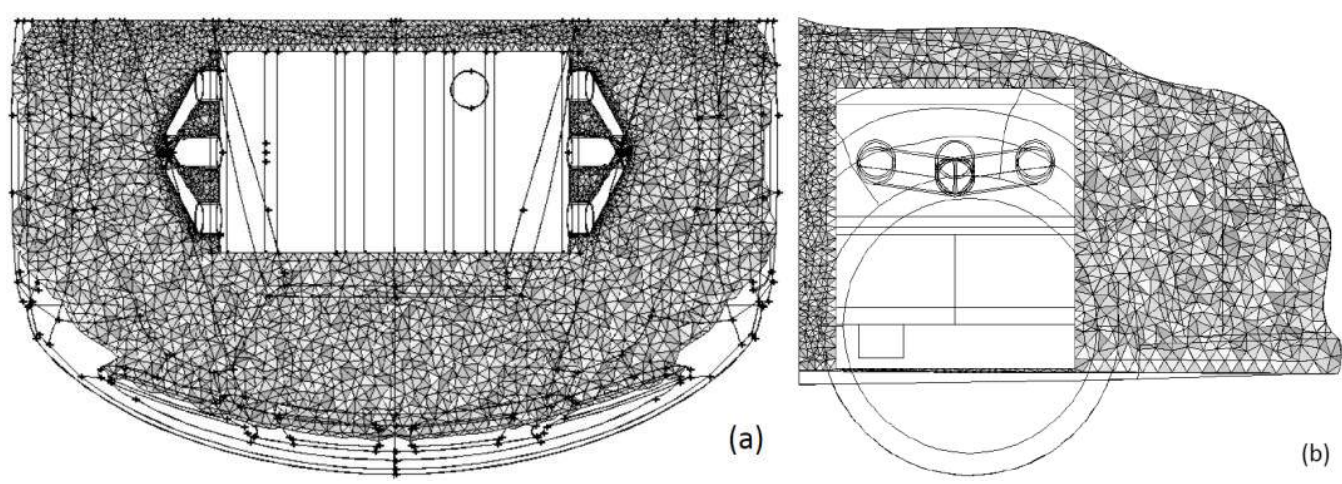

Figure 3. Mesh distribution of the underhood region taken at (a) horizontal plane and (b) vertical plane 


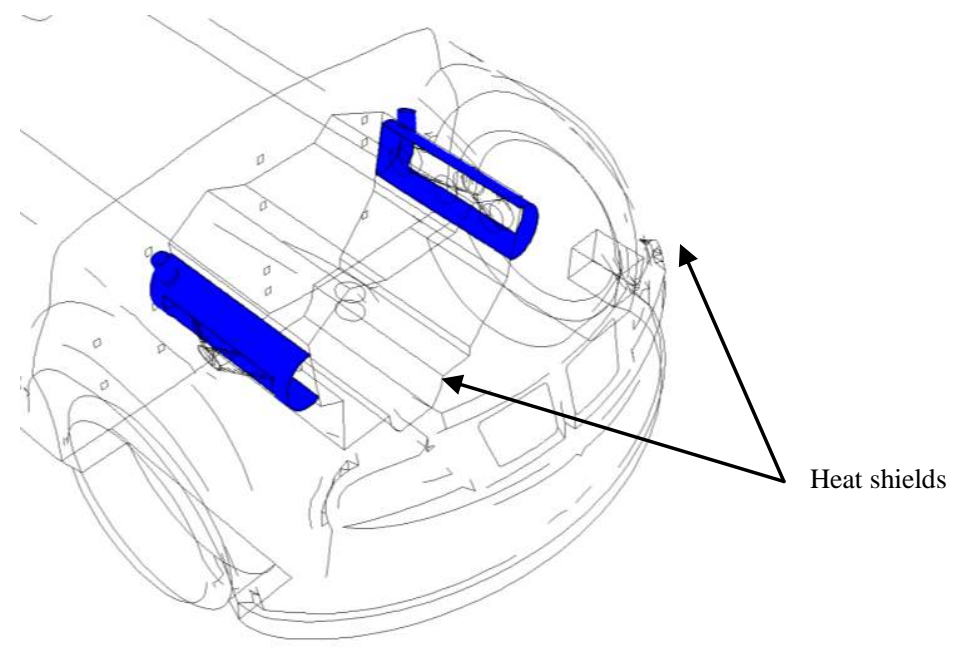

Figure 4. Proposed heat shield design located on exhaust manifolds (HSD)

\section{Numerical Model and Boundary Conditions}

The commercial CFD code FLUENT was used for the simulations. In brief, FLUENT applies a standard Finite Volume Method to solve the governing continuity, momentum and energy equations. For the current problem, pressure-velocity coupling was achieved using the SIMPLE algorithm, available as standard in FLUENT (ANSYS Inc., 2010). For turbulence modeling, the k- $\varepsilon$ turbulent model with wall function is used, whereby the turbulence kinetic energy, $k$, of the airflow is calculated with Eq. (1), and turbulence dissipation, $\varepsilon$, calculated from Eq. (2) as:

$$
\begin{gathered}
\frac{\partial k}{\partial t}+\frac{\partial}{\partial x_{i}}\left(\rho k u_{i}\right)=\frac{\partial}{\partial x_{j}}\left[\left(\mu+\frac{\mu_{t}}{\sigma_{k}}\right) \frac{\partial k}{\partial x_{j}}\right]+\rho v_{t} G-\rho \\
\frac{\partial}{\partial t}(\varepsilon)+\frac{\partial}{\partial x_{i}}\left(\rho \varepsilon u_{i}\right)=\frac{\partial}{\partial x_{j}}\left[\frac{\left(\mu+\frac{\mu_{t}}{\sigma_{\varepsilon}}\right) \partial \varepsilon}{\partial x_{j}}\right]+C_{1 \epsilon} \rho v_{t} G \frac{\varepsilon}{k}-C_{2 \varepsilon} \rho \frac{\varepsilon^{2}}{k}
\end{gathered}
$$

The inlet and outlet boundary conditions are applied, as shown in Figure 1, and all other surfaces are designated as walls. The inlet air velocity is set from $5-25 \mathrm{~m} / \mathrm{s}$, simulating a travelling car at $18-90 \mathrm{~km} / \mathrm{h}$, and the outlet boundary condition is set to an atmospheric pressure value. For the thermal analysis investigation, various factors need to be considered, such as the conditions of the underhood, as well as the heat transfer and material properties. By assuming the cooling system will maintain both the heat source at the optimum operating condition, the engine components and underhood experience constant heat from the engine block and exhaust manifolds. Constant surface temperatures of $365 \mathrm{~K}$ and $673 \mathrm{~K}$ are assigned to the engine block and exhaust manifolds, respectively. Both these temperatures are referred to as the average operating temperatures.

For the thermal calculations, a planar conduction model in FLUENT is used to account for the conduction characteristics inside each underhood component, which required the material and component thicknesses to be specified. Table 1 shows the boundary conditions defined for each component inside the underhood. For the radiation heat transfer calculations, the discrete ordinate method was selected due to its 
robustness for all optical thicknesses and its enhanced accuracy. The DO radiative transfer equation is given by Eq. (3) (Fellague et al., 1994):

$$
\frac{\partial I}{\partial x_{i}}+(I(\vec{r}, \vec{s}) \vec{s})+\left(a+\sigma_{s} I(\vec{r}, \vec{s})=a n^{2} \frac{\sigma T^{4}}{\pi}+\frac{\sigma_{s}}{4 \pi} \int_{0}^{4 \pi} I(\vec{r}, \vec{s}) \Phi\left(\vec{s}, \vec{s}^{\prime}\right) d \Omega^{\prime}\right.
$$

Internal emissivity at the boundary walls is set according to the material used and the wall temperature. Table 2 shows the emissivity values used for the radiation calculations.

Table 1. Boundary conditions for each underhood component

\begin{tabular}{llll}
\hline Components & Material & Thickness & Temperature \\
\hline $\begin{array}{l}\text { Engine } \\
\text { Exhaust }\end{array}$ & Steel & $0.5 \mathrm{~cm}$ & $365 \mathrm{~K}$ \\
manifolds & Steel & $0.4 \mathrm{~cm}$ & $673 \mathrm{~K}$ \\
Battery & Aluminum & $0.1 \mathrm{~cm}$ & $0 \mathrm{~W} / \mathrm{m}^{2}$ \\
Oil filter & Aluminum & $0.1 \mathrm{~cm}$ & $0 \mathrm{~W} / \mathrm{m}^{2}$ \\
Firewall & Steel & $0.3 \mathrm{~cm}$ & Coupled \\
Car wall & Steel & $0.2 \mathrm{~cm}$ & Coupled \\
Heat shield & Steel & $1.5 \mathrm{~mm}$ & Coupled \\
\hline
\end{tabular}

Table 2. Internal emissivity at wall boundaries

\begin{tabular}{lc}
\hline \multicolumn{1}{c}{ Boundary } & Internal Emissivity \\
\hline Engine block & 0.22 \\
Exhaust manifold & 0.25 \\
Firewall, Hood wall, Heat shield & 0.22 \\
Battery, Oil filter & 0.04 \\
\hline
\end{tabular}

\section{RESULTS AND DISCUSSION}

Figure 5 shows the comparisons of the temperature contours on the hood surface and the underhood region, between the BD and the cases with HSD. As expected, Figure 5(a) and (b) show that the addition of the heat shield has managed to lower the peak temperatures at the hood. In addition, the firewall temperature reduction in the HSD case is evident by comparing Figures 5(c) and 5(d). A more useful insight into the underhood flow and thermal behavior can be gained by looking at the different engine components within the underhood. In the following subsections, comparisons of the peak temperature profiles for three components (firewall, hood and battery) between the BD and HSD are used to compare the cooling efficiency between the two designs. As shown in Figure 6, the HSD has achieved the objective of lowing the temperature at the firewall. By introducing a heat shield, the temperature at the firewall is quite constant at around $311 \mathrm{~K}$, indicating the low dependency of the HSD on air velocity. The heat shield serves as an insulator for the firewall from the intense heat at the exhaust manifold. As expected, the heat shield blocks and channels the hot air away from the region around the firewall, ensuring a more consistent thermal distribution at the firewall. 

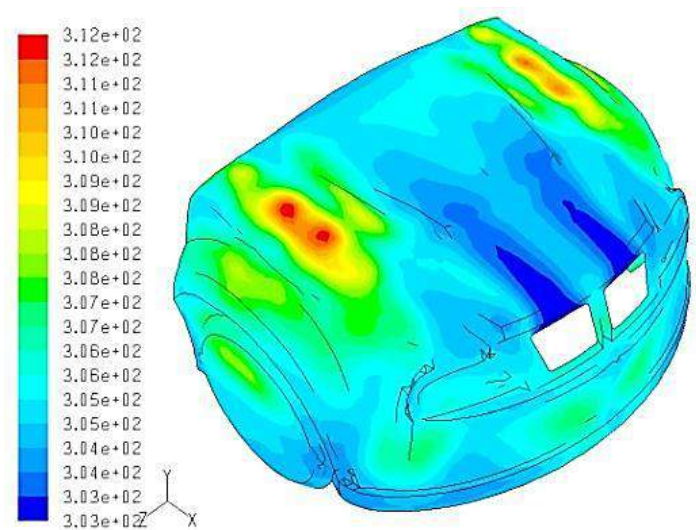

(a)

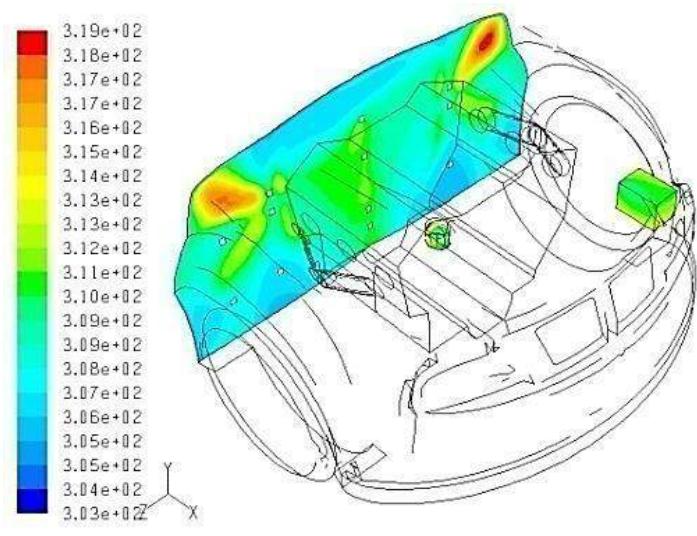

(c)

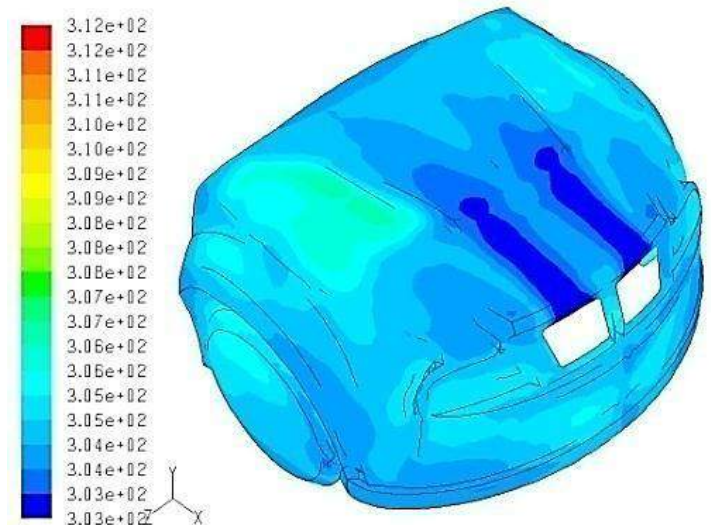

(b)

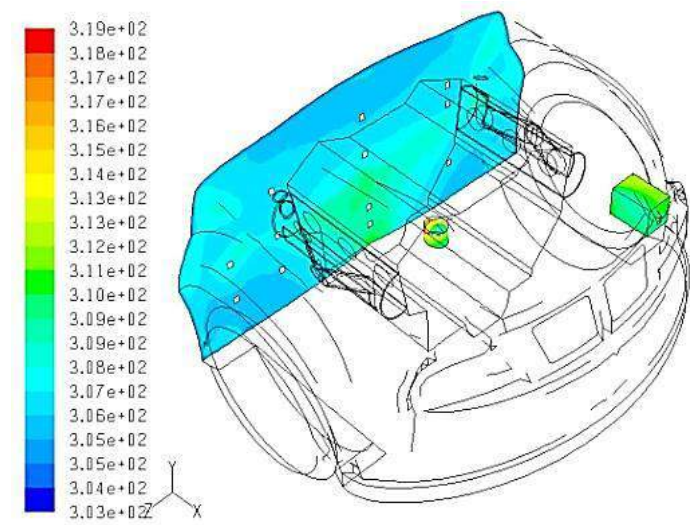

(d)

Figure 5. Temperature contour plots at $\mathrm{v}=15 \mathrm{~m} / \mathrm{s}$ for (a) Hood (BD), (b) Hood (HSD), (c) Underhood (BD), (d) Underhood (HSD)

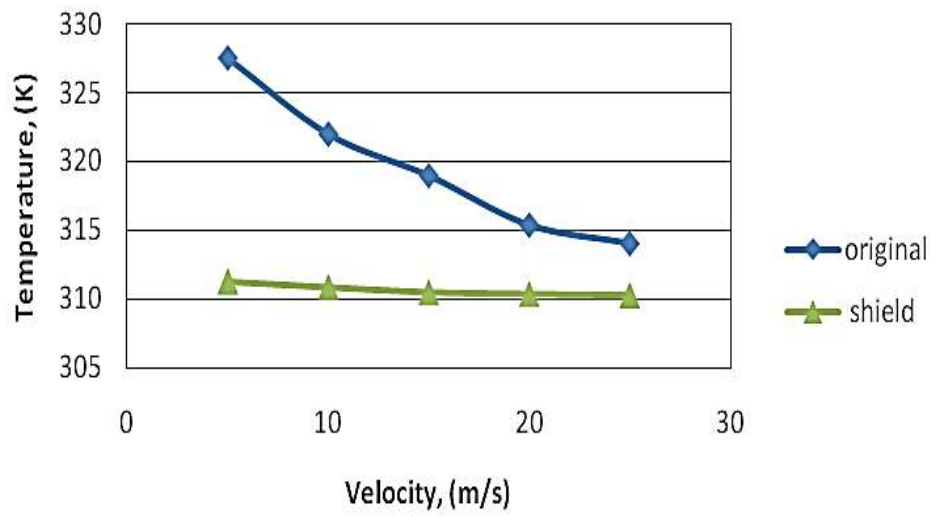

Figure 6. Peak temperatures of the firewall at different velocities: Comparison between BD and HSD

From the vector plot of the BD shown in Figure 7, it is seen that the flow moves across the heat sources towards the firewall, and moves sideways upon hitting the firewall, resulting in a backflow towards the front of the underhood. This backflow 
meets with the incoming inlet flow, which then forms a vortex of hot air at the frontal region of underhood. The heat shield inclusion ensures that the heated air is channeled in a more organized fashion. As shown in Figure 8, the heated air is channeled upwards and away from exhaust manifold, and this will prevent the heated air from being driven towards the firewall.

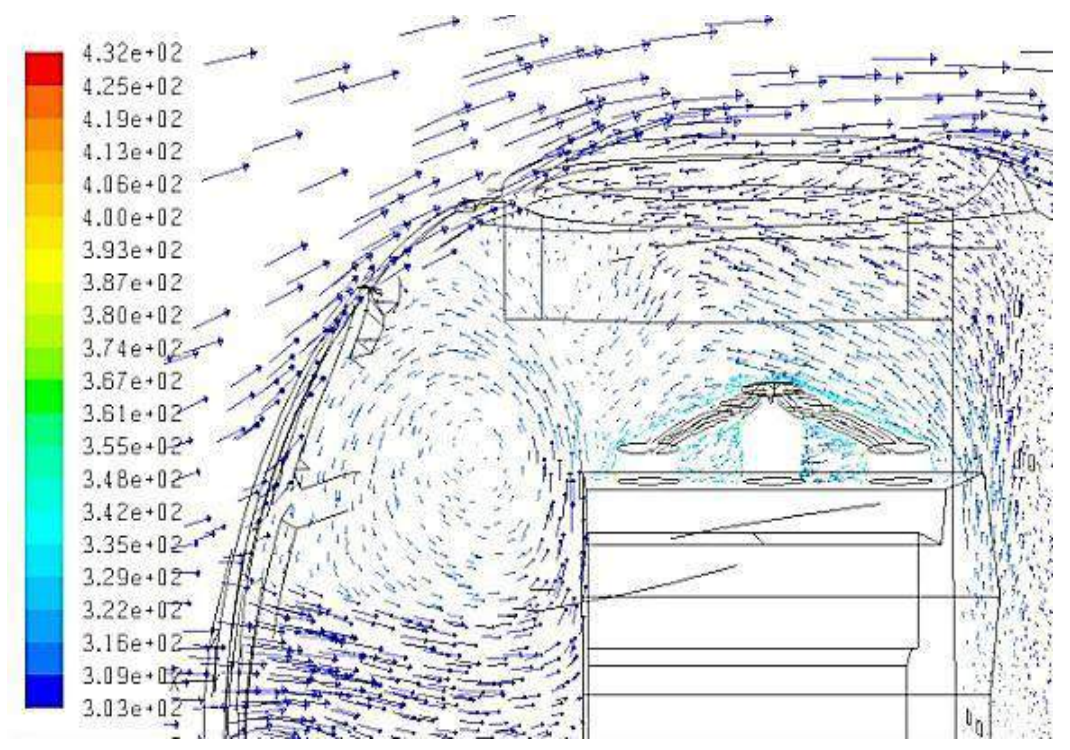

Figure 7. Velocity vector plot for BD (top view)

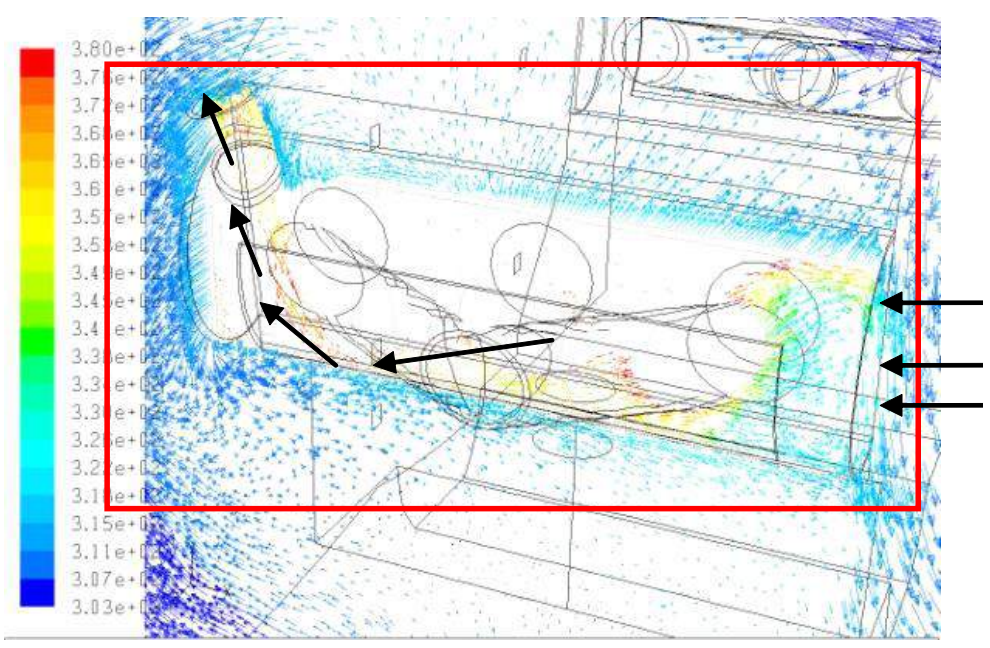

Figure 8. Velocity vector plot across the heat shield

Figure 9 shows the peak temperature at the hood surface for BD and HSD at different velocities. Again, the HSD results in a lower temperature profile compared to the BD. In particular, insulation as a result of the heat shield at low velocity is shown to significantly reduce the temperature of the underhood. At $5 \mathrm{~m} / \mathrm{s}$, the temperature drop for HSD at the hood is $9 \mathrm{~K}$, and as the velocity increases to $25 \mathrm{~m} / \mathrm{s}$ the temperature drop reduces to $2 \mathrm{~K}$. This is due to an increment in air turbulence in the underhood as the velocity increases, which in turn increases the heat dissipation and reduces the insulation effect of the heat shield. 


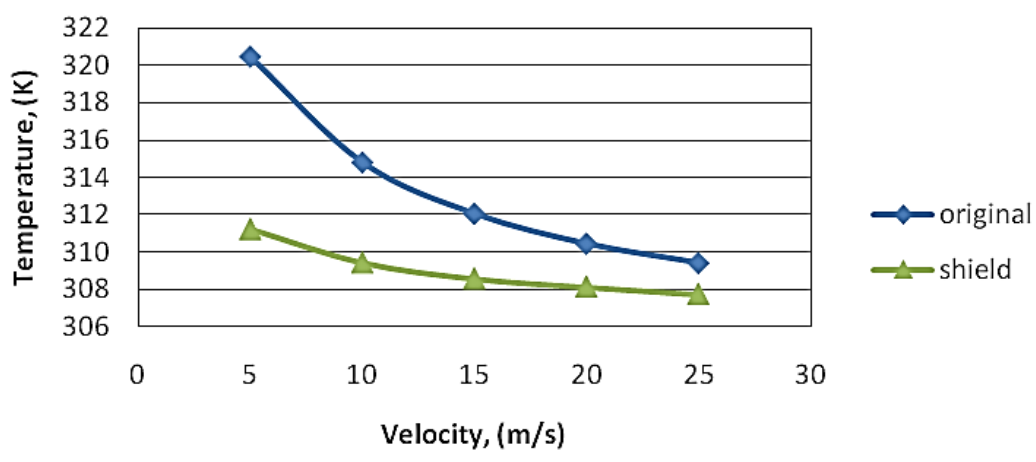

Figure 9. Peak temperatures of the hood surface at different velocities: Comparison between BD and HSD

As shown in Figure 10, the heat shield has in general managed to reduce the temperature of the battery, indicating an improvement in the heat distribution at the frontal region of underhood. The temperature at the battery in the HSD is $5 \mathrm{~K}$ lower than BD, and the temperature difference remains constant until around $15 \mathrm{~m} / \mathrm{s}$. From 20 $\mathrm{m} / \mathrm{s}$ onwards, the drop in temperature for BD is higher, and at $25 \mathrm{~m} / \mathrm{s}$ the peak temperature at the battery equals the HSD case.

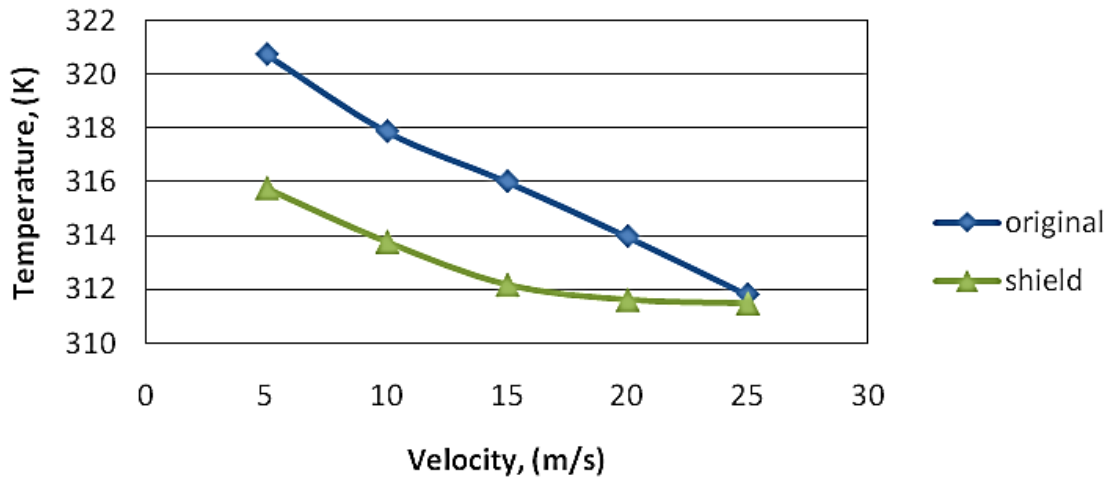

Figure 10. Peak temperatures of the battery at different velocities: Comparison between BD and HSD.

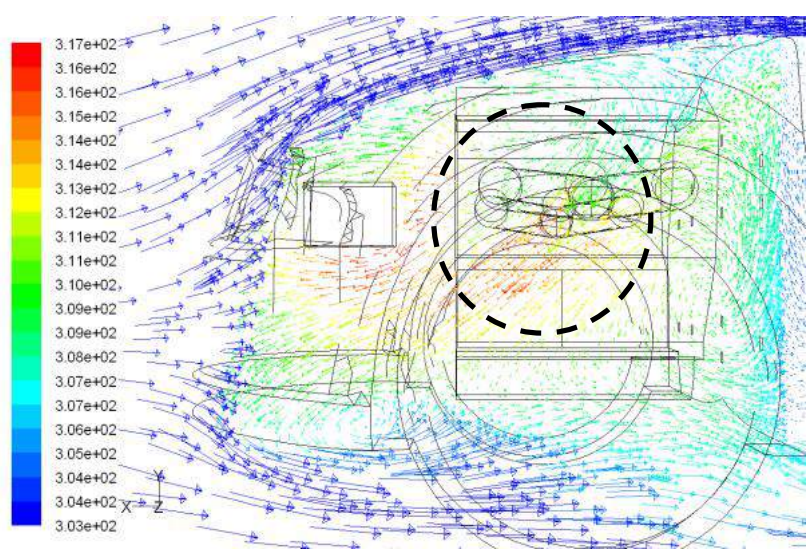

Figure 11 . Vector plot colored by temperature across battery at velocity of $15 \mathrm{~m} / \mathrm{s}$ (BD). 
As shown in Figure 11, investigation conducted for the BD at $15 \mathrm{~m} / \mathrm{s}$ shows that the heated air splits during the backflow and the battery experiences a direct hit of turbulence from the incoming heated air. With the addition of a heat shield, the heated air is prevented from flowing across the battery and is only channeled towards the underhood opening, as shown in Figure 12. Besides which, the air temperature flowing across the battery in the HSD is relatively lower. This has proven that the HSD can be a good measure to prevent intense heat from flowing towards the frontal region of the underhood, where most of the components made of materials with low melting points are located.

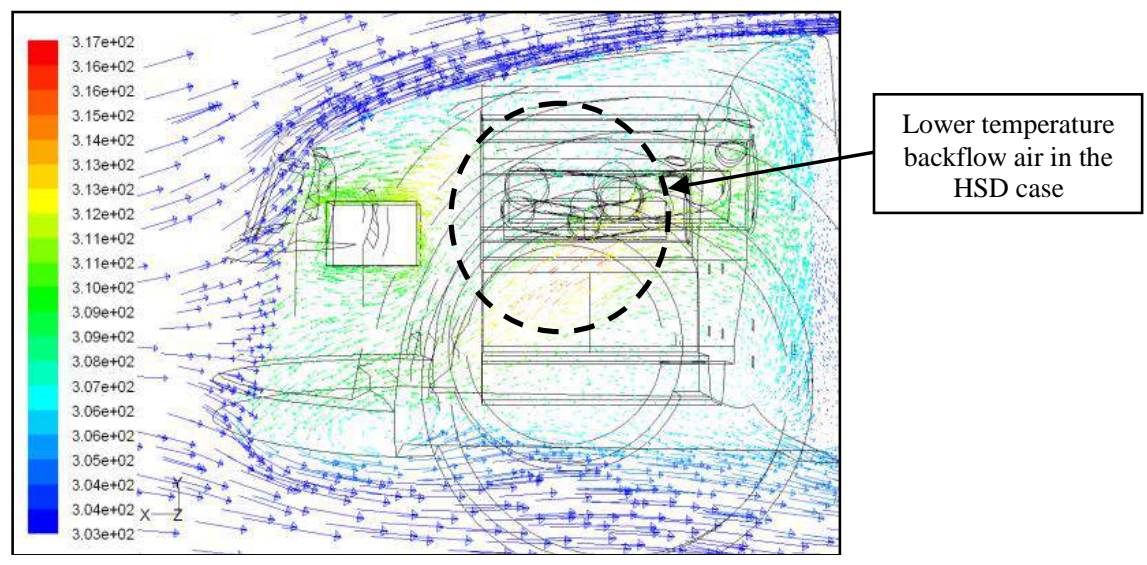

Figure 12 . Vector plot colored by temperature across the battery at a velocity of $15 \mathrm{~m} / \mathrm{s}$ (HSD)

\section{CONCLUSION}

In this study it was found that the proposed heat shield design can act as a good measure in improving the heat distribution and cooling performance on engine components, regardless of its positioning. A heat shield not only serves as an insulator, but also helps to regulate the air flow in the underhood. Simulation results show that the distribution of heated air in the underhood is more regulated as the heat from the heat source is channeled in a more appropriate manner, resulting in lower peak temperatures for various underhood components. The study shows the potential of the CFD method in optimizing the cooling processes in the underhood, as heat dissipation and air circulation within the underhood can be designed out.

\section{REFERENCES}

Amodeo, J., Alajbegovic, A. and Jansen, W. 2006. Thermal management simulation for passenger cars - towards total vehicle analysis. Exa Corporation and Jaguar Cars.

ANSYS Inc. 2010. FLUENT 12 User Manual. ANSYS Inc.

Campos, F., Geremia, P. and Weston, S. 2007. Fast robust design optimisation of an underhood cooling duct. NAFEMS World Congress, Vancouver, Canada.

Damodaran, V. and Kaushik, S. 2000. Simulation to identify and resolve underhood/ underbody vehicle thermal issue. Fluent Inc., pp. 1-4. 
Davis, F.V., Veling, T.R., Caltrider, J.L. and Madhavar, R. 1993. Impact of computer aided engineering on Ford motor company light truck cooling design and development processes. SAE Paper 93-2977.

Fellague, K.A., Hu, H. and Willoughby, D.A. 1994. Determination of the effects of inlet air velocity and temperature on the performance of an automotive radiator. SAE Paper 94-0771.

Hsu, I.F. and Schwartz, W.S. 1995. Simulation of the thermal environment surrounding an under-body fuel tank in a passenger vehicle using orthogonally structured and body fitted unstructured CFD codes in series. SAE Paper 95-0616.

Koschan, A., Govindasamy, P. Sukumar, S., Page, D. and Abidi, M. 2006. Thermal modeling and imaging of as-built vehicle components. SAE Technical Paper 2006-01-1167.

Srinivasan, K., Jan, J.Y., Sun, R.L. and Gleason, M.E. 1999. Rapid simulation methodology for under-hood aero/thermal management. International Journal of Vehicle Design, 23 (1-2): 109-123.

Srivanesan, K., Wang, Z.J., Yuan, W. and Sun, R. 2004. Vehicle thermal management simulation using a rapid omni-tree based adaptive cartesian mesh generation methodology. ASME Heat Transfer/Fluids Engineering Summer Conference, HT-FED2004-56748.

Wang, D. and Yang, Z. 2006. CFD predictions of minivan's front end flow performance. IEEE International Conference on Vehicular Electronics and Safety, Tongji University, Shanghai, 13-15 Dec., 1-4244-0759-1/06

Yang, Z., Bozeman, J. and Shen, F.Z. 2004. CFD for flow rate and air recirculation at vehicle idle condition. SAE Paper 2004-HX21. 